\title{
Early Adolescent MK-801 Exposure Impairs the Maturation of Ventral Hippocampal Control of Basolateral Amygdala Drive in the Adult Prefrontal Cortex
}

\author{
Daniel R. Thomases, Daryn K. Cass, Jacqueline D. Meyer, Adriana Caballero, and Kuei Y. Tseng \\ Department of Cellular and Molecular Pharmacology, The Chicago Medical School, Rosalind Franklin University of Medicine and Science, North Chicago, \\ Illinois 60064
}

\begin{abstract}
The adolescent susceptibility to the onset of psychiatric disorders is only beginning to be understood when factoring in the development of the prefrontal cortex (PFC). The functional maturation of the PFC is dependent upon proper integration of glutamatergic inputs from the ventral hippocampus (vHipp) and the basolateral amygdala (BLA). Here we assessed how transient NMDAR blockade during adolescence alters the functional interaction of vHipp-BLA inputs in regulating PFC plasticity. Local field potential recordings were used to determine changes in long-term depression (LTD) and long-term potentiation (LTP) of PFC responses resulting from vHipp and BLA high-frequency stimulation in adult rats that received repeated injections of saline or the NMDAR antagonist MK-801 from postnatal day 35 (P35) to P40. We found that early adolescent MK-801 exposure elicited an age- and input-specific dysregulation of vHipp-PFC plasticity, characterized by a shift from LTD to LTP without altering the BLA-induced LTP. Data also showed that the vHipp normally resets the LTP state of BLA transmission; however, this inhibitory regulation is absent following early adolescent MK-801 treatment. This deficit was reminiscent of PFC responses seen in drug-naive juveniles. Notably, local prefrontal upregulation of $\mathrm{GABA}_{\mathrm{A}} \alpha 1$ function completely restored vHipp functionality and its regulation of BLA plasticity in MK-801-treated rats. Thus, NMDAR signaling is critical for the periadolescent acquisition of a GABA-dependent hippocampal control of PFC plasticity, which enables the inhibitory control of the prefrontal output by the vHipp. A dysregulation of this pathway can alter PFC processing of other converging afferents such as those from the BLA.
\end{abstract}

Key words: adolescence; GABA; interneurons; NMDA; plasticity

\section{Introduction}

The transition from adolescence to adulthood represents a window of enhanced vulnerability for the onset of major psychiatric disorders, including schizophrenia, depression, and substance abuse (Kessler et al., 2007; Paus et al., 2008; Gogtay et al., 2011). The precise neurobiology underlying this susceptibility is only beginning to be understood when factoring in the development of the prefrontal cortex (PFC), a key structure for sustaining higher cognitive functions whose maturation occurs during adolescence (Casey et al., 2000; Best and Miller, 2010). Indeed, the onset of such disorders is often associated with impairments of a variety of PFC-dependent cognitive processes (Lewis et al., 2012), many of which require proper integration of glutamatergic transmission from the ventral hippocampus (vHipp) and the basolat-

\footnotetext{
Received April 7, 2014; revised May 24, 2014; accepted May 29, 2014.

Author contributions: D.R.T. and K.Y.T. designed research; D.R.T., D.K.C., and J.D.M. performed research; D.R.T. and K.Y.T. analyzed data; D.R.T., A.C., and K.Y.T. wrote the paper.

This research was supported by Rosalind Franklin University of Medicine and Science and National Institutes of Health Grant R01-MH086507 to K.Y.T.

Correspondence should be addressed to Dr. Kuei Y. Tseng, Department of Cellular and Molecular Pharmacology, The Chicago Medical School, Rosalind Franklin University of Medicine and Science, 3333 Green Bay Road, North Chicago, IL 60064. E-mail: kuei-yuan.tseng@rosalindfranklin.edu.

DOI:10.1523/JNEUROSCI.1395-14.2014

Copyright $\odot 2014$ the authors $\quad 0270-6474 / 14 / 349059-08 \$ 15.00 / 0$
}

eral amygdala (BLA) (Phillips and LeDoux, 1992; Ishikawa and Nakamura, 2003; Sierra-Mercado et al., 2011; Gilmartin et al., 2012; Sotres-Bayon et al., 2012), two limbic structures that project heavily to the PFC (Ishikawa and Nakamura, 2003; Tierney et al., 2004; Gabbott et al., 2006). Thus, a proper understanding of normal PFC function will require knowledge of how these inputs interact to shape PFC output, and if so, how a developmental interference during adolescence could result in maladaptive PFC processing in adulthood.

We have recently determined that inputs arising from the vHipp and the BLA elicit distinct forms of long-term plasticity in the PFC in an age-dependent manner (Caballero et al., 2014; Flores-Barrera et al., 2014). In particular, ventral hippocampal stimulation produces a GABA-dependent form of plasticity in the PFC that does not emerge until late adolescence (Caballero et al., 2014; Flores-Barrera et al., 2014). In contrast, the response elicited from the BLA is already present in the adolescent PFC and does not require local GABAergic transmission (Caballero et al., 2014). Although the cellular mechanism underlying such inputand age-specific regulation of PFC afferent processing remains unclear, our recent study indicates that the functional maturation of the vHipp-PFC connectivity is activity dependent and requires sustained NMDAR-mediated glutamatergic transmission throughout adolescence (Thomases et al., 2013). Given the critically inter- 
a

posnatal days

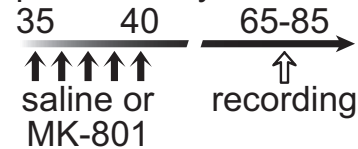

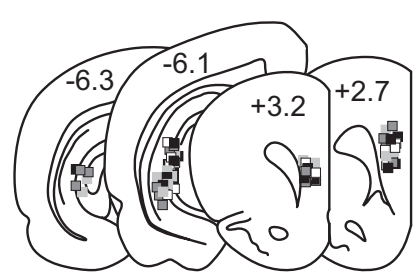

e

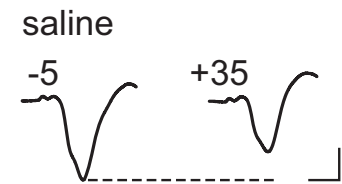

b

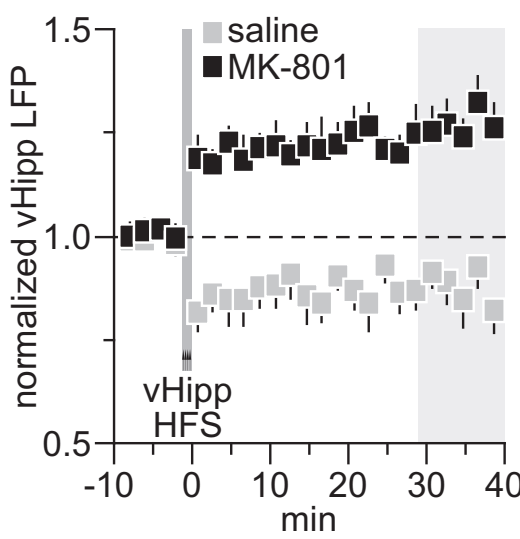

C

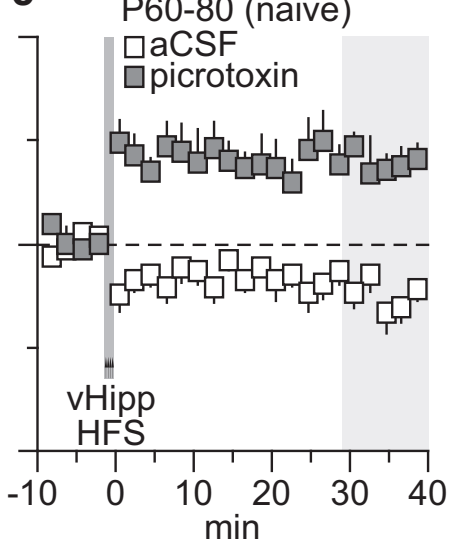

d

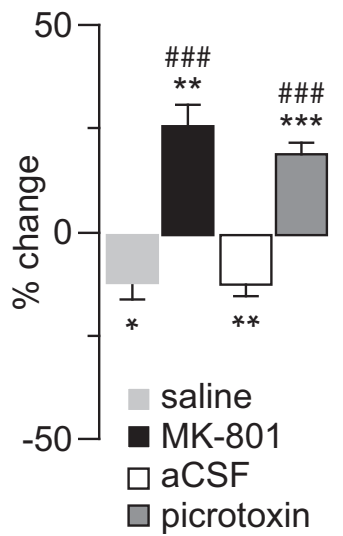

MK-801

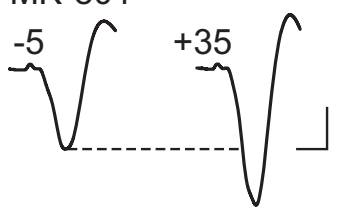

P60-80 (aCSF)

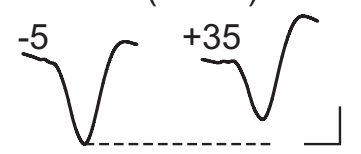

P60-80 (picrotoxin)

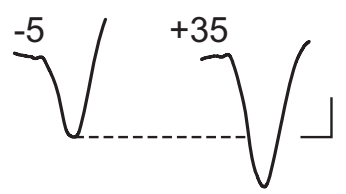

Figure 1. Disruption of vHipp-induced plasticity in the PFC by early adolescent MK-801 exposure. $\boldsymbol{a}$, Timeline of the experimental design and summary of the recording sites (PFC: +3.2 to +2.7 $\mathrm{mm}$ from bregma) and stimulating sites (vHipp: -6.1 to $-6.3 \mathrm{~mm}$ from bregma). $\boldsymbol{b}$, PFC LFP recordings from adult rats (P65-P85) that received saline $(n=10)$ or MK-801 ( $n=9$ ) treatment during

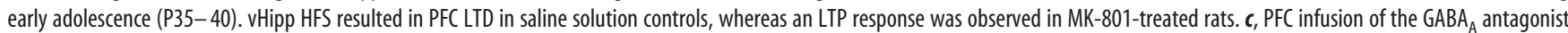
picrotoxin $(50 \mu \mathrm{m} / 1 \mu \mathrm{l} ; n=11)$ was sufficient to shift the normal vHipp-induced LTD (aCSF, $n=7)$ to LTP in adult naive rats (P60 - 80). $\boldsymbol{d}$, Summary of the mean LFP response obtained at 30 - 40 min post-HFS. ${ }^{*} p<0.05,{ }^{* *} p<0.005,{ }^{* * *} p<0.0005$ vs baseline, paired $t$ test. Note that the magnitude of PFC LTP observed in MK-801-treated rats resembles that induced by local PFC infusion of picrotoxin (\#\#\# $p<0.0005$ vs saline solution or aCSF, LSD post hoc test after significant one-way ANOVA; $\left.F_{(3,33)}=28.9 ; p<0.0001\right)$. e, Example traces of the vHipp-evoked PFC response taken from 5 min pre-HFS ( -5$)$ and 35 min post-HFS ( +35$)$. Calibration: $4 \mathrm{mV}, 20 \mathrm{~ms}$.
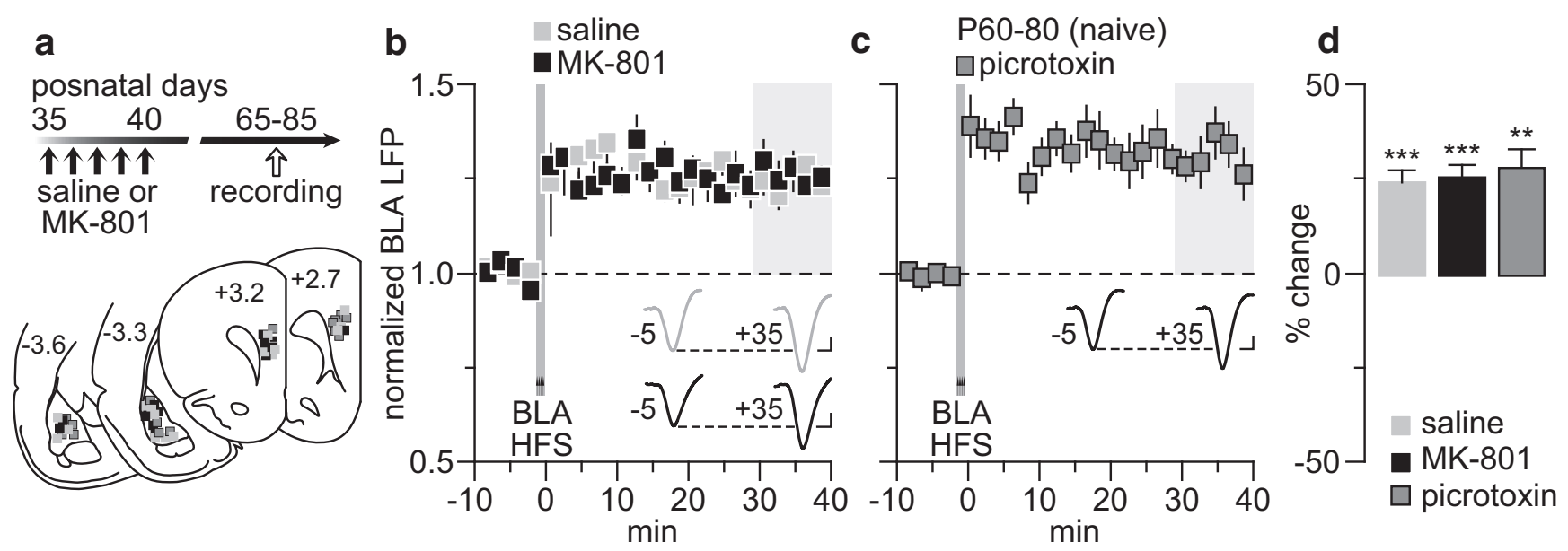

Figure 2. BLA-induced plasticity in the PFC remained unaltered by the early adolescent MK-801 treatment. $\boldsymbol{a}$, Timeline of the experimental design and summary of the recording sites (PFC: +3.2 to $+2.7 \mathrm{~mm}$ from bregma) and stimulating sites (BLA: -3.3 to $-3.6 \mathrm{~mm}$ from bregma). $\boldsymbol{b}$, PFC LFP recordings from adult rats that received saline $(n=9)$ or MK-801 ( $n=9)$ treatment during early adolescence (P35-40). HFS of the BLA resulted in PFC LTP in both saline- and MK-801-treated groups. C, PFC infusion of picrotoxin $(50 \mu \mathrm{m} / 1 \mu \mathrm{l} ; n=8)$ failed to affect the BLA-induced LTP in adult naive rats (P60-80). $\boldsymbol{d}$, Summary of the mean LFP response obtained at $30-40$ min post-HFS. ${ }^{* *} p<0.005,{ }^{* * *} p<0.0005$ vs baseline, paired $t$ test. Insets in $\boldsymbol{b}$ and $\boldsymbol{c}$ are example traces of the BLA-evoked PFC response taken from $5 \mathrm{~min}$ pre-HFS ( -5 ) and $35 \mathrm{~min}$ post-HFS ( +35 ). Calibration: $4 \mathrm{mV}, 20 \mathrm{~ms}$.

connected roles of the vHipp and the BLA in mediating PFC functions (Ishikawa and Nakamura, 2003; Sierra-Mercado et al., 2011; Sotres-Bayon et al., 2012; Caballero et al., 2014), it is possible that a similar activity-dependent susceptibility extends to the BLA-to-PFC pathway. Thus, a more pressing question is whether transient developmental disruptions occurring during adolescence could permanently alter the balance of vHipp and BLA regulation of PFC plasticity and diminish the functional capacity of prefrontal output.
In the present study, we conducted local field potential (LFP) recordings of afferent-evoked responses and examined how transient blockade of NMDARs during early adolescence affects the interaction of the vHipp and BLA drives in the PFC. More specifically, rats received repeated noncontingent injections of the noncompetitive NMDAR antagonist MK-801 from postnatal day 35 (P35) to P40 (Thomases et al., 2013), and changes in BLA-induced PFC plasticity and its heterosynaptic modulation by vHipp input and local prefrontal GABAergic transmission were determined in adulthood (P65-85). 

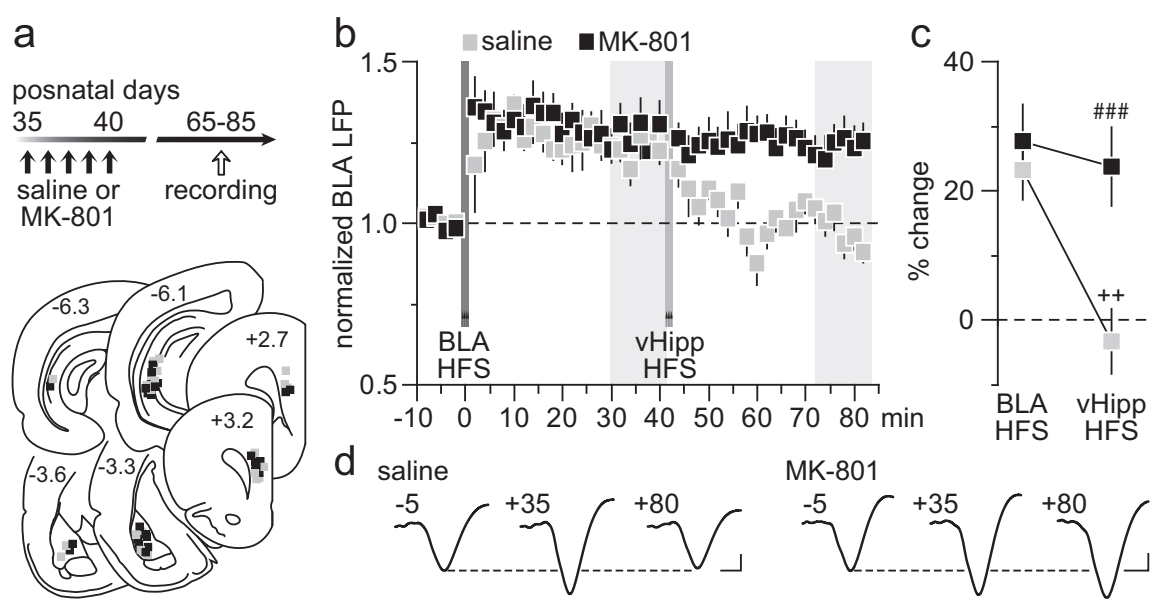

Figure 3. Early adolescent MK-801 exposure impaired the normal vHipp-dependent depotentiation of BLA-evoked responses in the PFC. $\boldsymbol{a}$, Timeline of the experimental design and summary of the recording sites (PFC) and stimulating sites (vHipp and BLA). $\boldsymbol{b}$, Impact of vHipp HFS on BLA-induced LTP in the PFC recorded from adult rats (P65-85) that received saline $(n=8)$ or MK-801 $(n=9)$ treatment during early adolescence. Note that the normal vHipp-dependent depotentiation of BLA-LTP is lacking in the PFC of MK-801-treated rats. C, Summary of the mean PFC response obtained at $30-40$ min post-HFS. Two-way ANOVA revealed a main effect of treatment $\left(F_{(1,30)}=10.4, p<0.005\right)$, a main effect of input stimulation $\left(F_{(1,30)}=9.7, p<0.005\right)$, and a treatment $X$ input stimulation interaction $\left(F_{(1,30)}=5.4, p<0.03\right.$; \#\#\# $<0.0005$ vs saline, $++p<0.005$ vs BLA HFS, LSD post hoc test). $\boldsymbol{d}$, Example traces of the BLA-evoked PFC response recorded at 5 min pre-BLA HFS $(-5), 35$ min post-BLA HFS $(+35)$, and 40 min post-vHipp HFS (+80). Calibration: $4 \mathrm{mV}, 20 \mathrm{~ms}$.

\section{a}

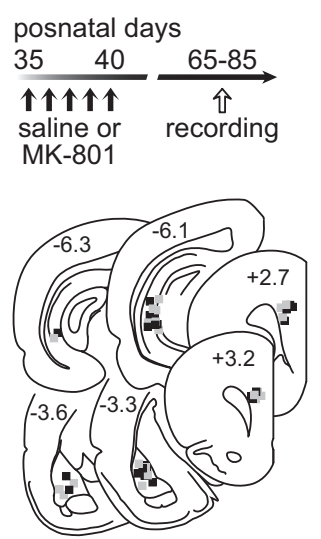

b

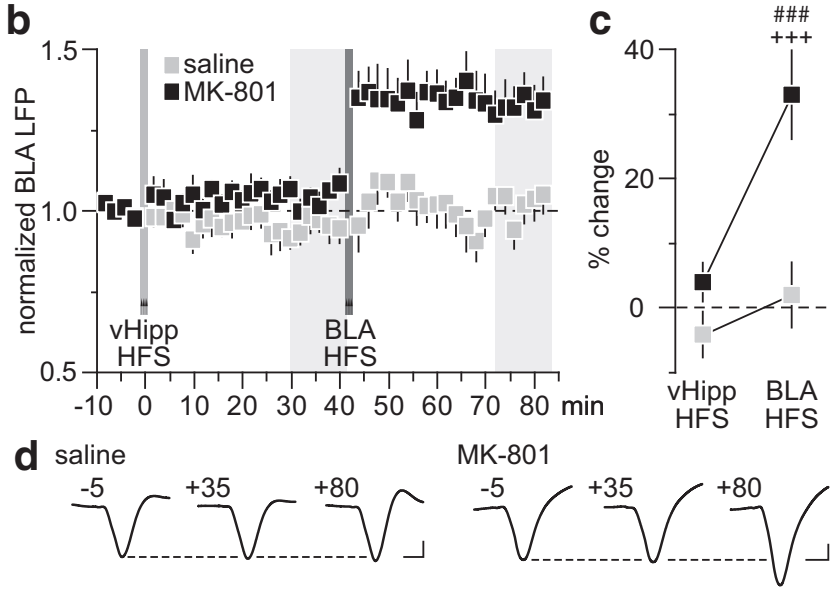

Figure 4. The normal vHipp-mediated inhibitory control of BLA-dependent plasticity in the PFC is lacking after early adolescent MK-801 treatment. $\boldsymbol{a}$, Timeline of the experimental design and summary of the recording sites (PFC) and stimulating sites (vHipp and BLA). $\boldsymbol{b}$, Impact of vHipp HFS before induction of BLA-dependent PFC LTP recorded from adult rats (P65-85) that received saline $(n=7)$ or MK-801 ( $n=7)$ treatment during early adolescence. Typically, vHipp HFS prevents the expression of BLA-induced LTP in the PFC of saline controls. Such inhibitory control by the vHipp drive is lacking in the MK-801-treated group. C, Summary of the mean PFC response obtained at 30-40 min post-HFS. Two-way ANOVA revealed a main effect of treatment $\left(F_{(1,24)}=16.1\right.$, $p<0.005)$, a main effect of input stimulation $\left(F_{(1,24)}=12.9, p<0.002\right)$, and a treatment $X$ input stimulation interaction

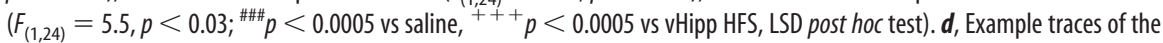
BLA-evoked PFC response recorded at 5 min pre-vHipp HFS $(-5), 35$ min post-vHipp HFS $(+35)$, and 40 min post-BLA HFS $(+80)$. Calibration: $4 \mathrm{mV}, 20 \mathrm{~ms}$.

\section{Materials and Methods}

All experimental procedures were approved by the Rosalind Franklin University of Medicine and Science Institutional Animal Care and Use Committee, and met the National Institutes of Health guidelines for the care and use of laboratory animals. Upon arrival, male Sprague Dawley rats (Harlan) were allowed to habituate for at least $5 \mathrm{~d}$ before being subjected to any experimental manipulation. In the present study, rats were group housed (two to three rats/cage), maintained under conditions of constant temperature $\left(21-23^{\circ} \mathrm{C}\right)$, and kept in a $12 \mathrm{~h}$ light/dark cycle with food and water available ad libitum. All chemicals were obtained from SigmaAldrich except for indiplon, which was purchased from Tocris Bioscience.

Experimental groups. Early adolescent (P35) rats were randomly assigned to receive daily noncontingent (home cage) intraperitoneal injections of saline solution $(0.9 \% \mathrm{NaCl})$ or MK-801 (0.1 mg/kg in saline solution) for 5 consecutive days as in our previous study (Thomases et al., 2013). Recordings were then conducted within the P65-P85 age range of adulthood (25-45 d after the last injection). In the adult-treated group (P75-P80), recordings were performed after an equivalent washout period from the last saline solution or MK-801 injection as in the early adolescent-treated rats.

In vivo recordings of LFPs in the medial PFC after ventral hippocampal and basolateral amygdala stimulation. All recordings were conducted in animals deeply anesthetized with $8 \%$ chloral hydrate (400 mg/kg, i.p.) as previously described (Thomases et al., 2013). Briefly, medial PFC LFP responses elicited from the vHipp and BLA were recorded using a concentric bipolar electrode (SNE-100x 50 mm; Rhodes Medical Instruments Inc.), amplified (Cygnus Technology Inc.), filtered (bandwidth, 1-100 Hz), and digitized (Digidata 1440A; Molecular Devices) at a sampling rate of $10 \mathrm{kHz}$. For stimulation, concentric bipolar electrodes (NE-100x, $50 \mathrm{~mm}$ ) were lowered into the vHipp and BLA, and electrical pulses of $300 \mu$ s duration at 0.75 $\mathrm{mA}$ were delivered every $15 \mathrm{~s}$ through a computer-controlled pulse generator (Master-8 Stimulator; A.M.P.I.). Typically, a $20 \mathrm{~min}$ period of stable baseline recording of afferentevoked LFPs was collected in the PFC $(<15 \%$ variability in evoked LFP amplitude and slope) before a protocol of high-frequency stimulation (HFS; 50 pulses at $100 \mathrm{~Hz} / 15 \mathrm{~s} \times 4$; Caballero et al., 2014) was delivered into the vHipp or the BLA. Afferent-driven plasticity in the PFC was determined by measuring changes in the slope of the evoked LFP (measured from the onset to peak amplitude of the evoked LFP) within the $40 \mathrm{~min}$ post-HFS period. Here, a bin size window of $2 \mathrm{~min}$ (i.e., the mean slope value from eight evoked LFPs) was used to compute the time course data points of the evoked LFP response before and after the HFS. For local prefrontal modulation of GABAergic transmission, a 28 gauge infusion cannula (length, $11 \mathrm{~mm}$; Plastics One Inc.) was secured to the recording electrode to allow microinfusions $(0.1 \mu \mathrm{l} / \mathrm{min})$ of aCSF-containing picrotoxin (1 $\mu \mathrm{l}$ at $50 \mu \mathrm{M} / 0.1 \%$ DMSO), the $\mathrm{GABA}_{\mathrm{A}} \alpha 1-$ positive allosteric modulator indiplon $(0.6 \mu \mathrm{l}$ at $10 \mu \mathrm{M} / 0.04 \%$ DMSO; Petroski et al., 2006) or vehicle ( $1 \mu \mathrm{l}$ of $0.1 \%$ DMSO and $0.6 \mu \mathrm{l}$ of $0.04 \%$ DMSO, respectively). The exact location of the recording and stimulation sites was determined by means of Nissl staining, as previously described (Thomases et al., 2013).

Statistical analysis. All time course plots summarizing the effects of HFS-induced LFP changes were computed using a bin size window of 2 min each (i.e., average slope from eight evoked LFPs per data point). Differences among experimental conditions were considered statistically significant at $p<0.05$, and the data are summarized as the mean \pm SEM. The Student's $t$ test was used for two-group comparisons involving a single continuous variable, whereas ANOVA was 
used for comparing the effects along three or more variables (StatSoft).

\section{Results}

We first investigated whether vHippinduced PFC plasticity (Caballero et al., 2014) is impaired in rats with a history of early adolescent MK-801 exposure (Fig. 1a). Recordings conducted in adulthood revealed that the normative HFS-induced long-term depression (LTD) of hippocampus-evoked LFPs observed in saline controls is lacking in MK801-treated rats (Fig. 1b). Instead, a longterm potentiation (LTP) emerges in the MK-801-treated group (Fig. 1b). Interestingly, a switch from HFS LTD to LTP can be induced in the normal adult PFC (P60-P80) with recordings conducted after local infusion of the $\mathrm{GABA}_{\mathrm{A}}$ receptor antagonist picrotoxin (Fig. 1c). While aCSF alone did not alter the LTD response, the magnitude of the LTP resembled that found in the PFC of early adolescent MK-801-treated rats (Fig. $1 b, c)$. We next asked whether PFC plasticity elicited by BLA HFS (Caballero et al., 2014) is also compromised after early adolescent MK-801 treatment (Fig. 2a). Data indicate that BLA-dependent plasticity (i.e., LTP) in the PFC remains unaltered following early adolescent MK-801 treatment (Fig. 2b). Interestingly, this form of plasticity does not require local prefrontal GABAergic transmission (Fig. $2 c$ ) because the pattern and magnitude of LTP recorded in the presence of picrotoxin were indistinguishable from that seen in saline controls and MK-801treated rats (Fig. $2 d$ ). Together, these results point to a GABAergic-dependent, input-specific disruption of PFC plasticity by early adolescent MK-801 exposure that endures through adulthood.

The lack of vHipp-dependent LTD in the PFC resulting from early adolescent MK-801 exposure could lead to abnormal prefrontal processing of afferent information. To test this, we examined how HFS of the vHipp modulates BLA transmission, in particular after the BLA-induced LTP in the PFC (Fig. 3a). We found that the normal hippocampal HFSdependent depotentiation of BLA-induced LTP is lacking in the PFC of early adolescent MK-801-treated rats (Fig. 3b). While vHipp HFS effectively reset the prefrontal LTP driven by the BLA in saline controls, it failed to alter the potentiated BLA-evoked LFP in the MK-801-treated group (Fig. 3c). Similarly, a blockade of BLA-induced LTP was observed in the PFC of saline controls when vHipp HFS preceded that of the BLA (Fig. $4 a, b$ ). In contrast, HFS of the vHipp failed to prevent the expression of BLAinduced LTP in the PFC of MK-801-treated rats (Fig. 4b,c). Together, these results indicate that the vHipp drive to the PFC exerts a powerful control over that from the BLA, a form of plasticity that is lacking after early adolescent MK-801 exposure.
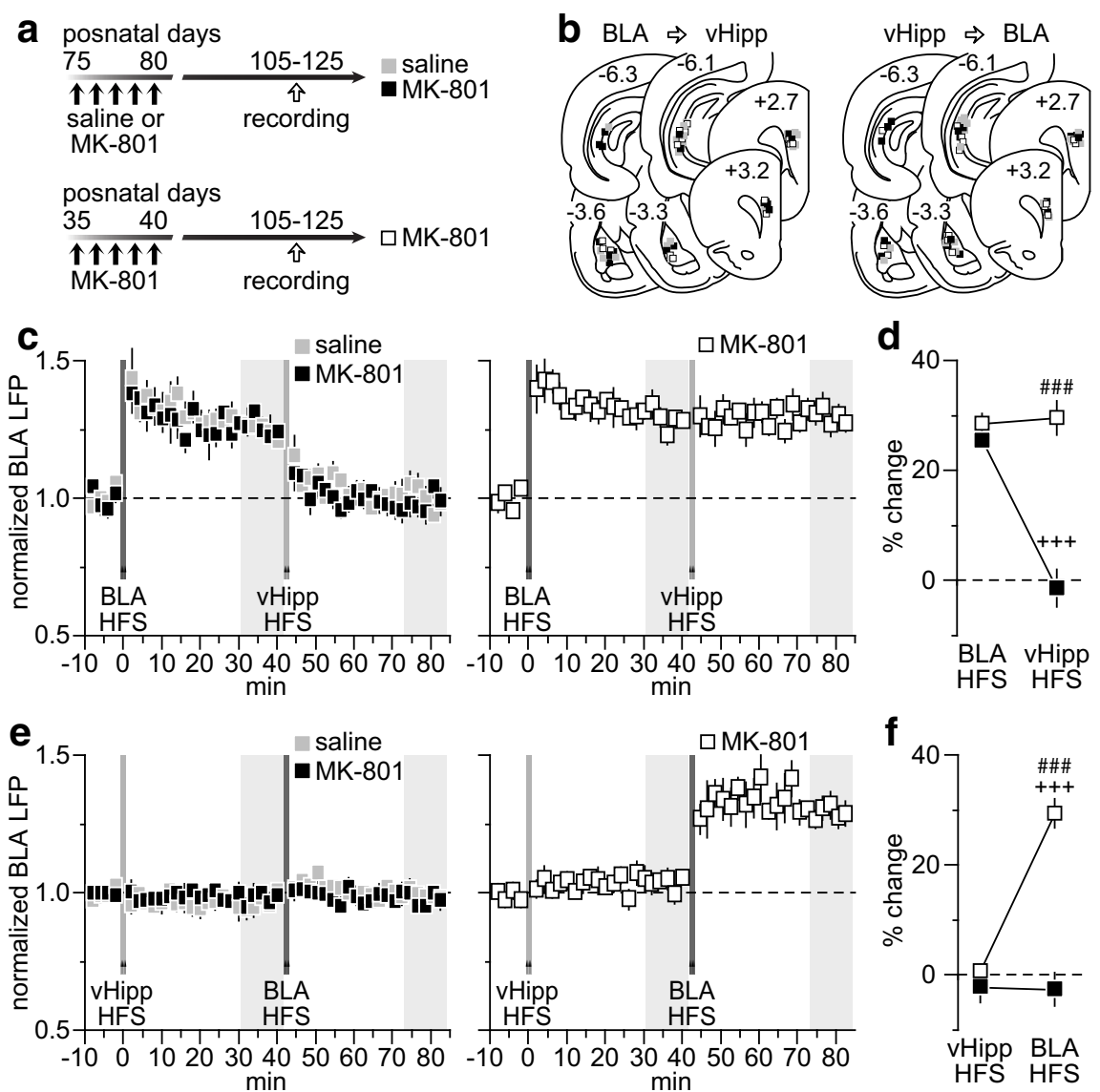

Figure 5. Impact of MK-801 treatment during adulthood on vHipp-dependent regulation of BLA transmission in the PFC. $\boldsymbol{a}$, Timeline of the experimental design. Note that another cohort of early adolescent MK-801-treated rats was included to control for the age of the recording period. $\boldsymbol{b}$, Summary of the recording sites (PFC) and stimulating sites (BLA and vHipp). $\boldsymbol{c}$, Relative to saline ats $(n=6)$. This was not the case in rats treated in early adolescence as the impaired vHipp-dependent depotentiation of - LTP resulting from MK-801 exposure (Fig. 3 ) endures through the P105-125 period $(n=5)$. $\boldsymbol{d}$, Summary of the mean PFC white) revealed a main effect of age of treatment $\left(F_{(1,18)}=42.1, p<0.0005\right)$, a main effect of input stimulation $\left(F_{(1,18)}\right.$ $24.6, p<0.0005)$, and an age of treatment $X$ input stimulation interaction $\left(F_{(1,18)}=28.6, p<0.0005 ;\right.$; $p<0.0005$ vs rats indicate that MK-801 exposure during adulthood did not impair the normal inhibitory control of the vHipp drive $(n=6$ per group). This was not the case for the early adolescent MK-801-treated group in which vHipp HFS failed to prevent the expression of BLA-LTP in the PFC, an impairment that endures through P105-125 period $(n=5)$. $f$, Summary of the mean PFC response revealed a main effect of age of treatment $\left(F_{(1,18)}=46.8, p<0.0005\right)$, a main effect of input stimulation $\left(F_{(1,18)}=30.4, p<\right.$ $0.0005)$, and an age of treatment $X$ input stimulation interaction $\left(F_{(1,18)}=31.8, p<0.0005\right.$; \#\#\# $p<0.0005$ vs adult treated, $+++p<0.005$ vs vHipp HFS, LSD post hoc test).

To determine whether the MK-801-induced disruption of vHipp regulation of BLA drive in the PFC is age dependent, we conducted recordings from rats that received MK-801 exposure during adulthood (i.e., P75-P80) following the same experimental design used in the early adolescent group. Thus, changes in LFP responses in the PFC were determined within 25-45 d from the last saline or MK-801 injection (i.e., P105-P125; Fig. 5a,b). We found that both saline- and MK-801-treated rats exhibited comparable levels of PFC LTP in response to BLA HFS (Fig. $5 c$ ). Interestingly, the normal vHipp-dependent depotentiation of the BLA response remained intact in both groups (Fig. 5c), indicating that transient NMDAR blockade during adulthood does not result in long-lasting deficits of hippocampal modulation, as seen in the early adolescent-treated group (Fig. 3). Importantly, the different postnatal age of the recording period 
a

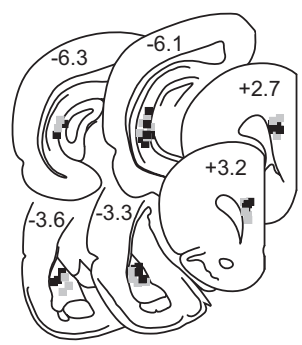

b

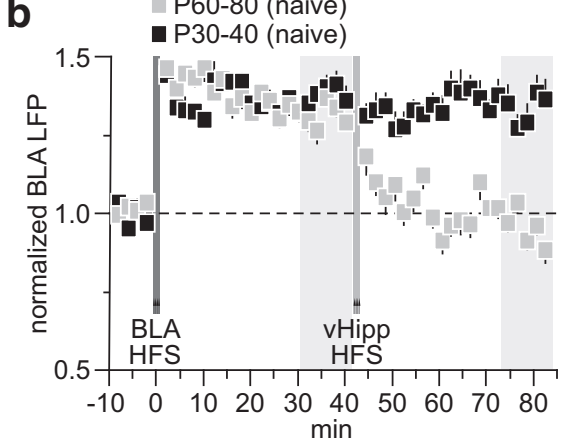

e

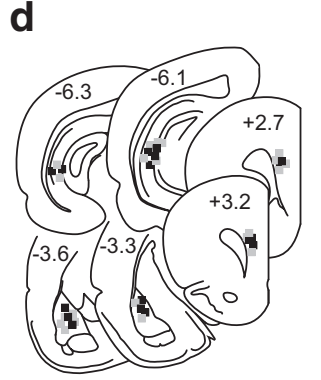

C
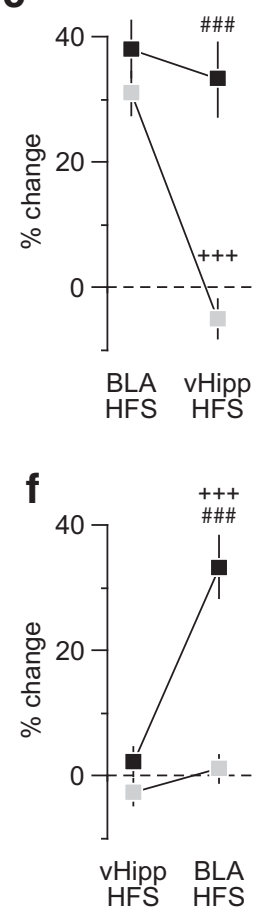

Figure 6. Developmental regulation of vHipp-dependent inhibitory control of BLA transmission in the PFC. $\boldsymbol{a}$, Summary of the recording sites (PFC) and stimulating sites (BLA and vHipp). $\boldsymbol{b}$, Age-dependent effect of vHipp HFS on BLA-LTP in the PFC. A clear depotentiation of the BLA-LTP by the vHipp drive was observed only in the P60 - 80 age group $(n=7)$, whereas HFS of the vHipp failed to alter the BLA-evoked LTP in the P30 - $40 \mathrm{PFC}(n=7)$. c, Summary of the PFC response obtained at $30-40$ min post-HFS. Two-way ANOVA revealed a main effect of input stimulation $\left(F_{(1,24)}=19.7, p<0.0005\right)$, a main effect of age $\left(F_{(1,24)}=28.4, p<\right.$ $0.0005)$, and an age $\times$ input stimulation interaction $\left(F_{(1,24)}=14.1, p<0.005 ; \# p<0.0005\right.$ vs $\mathrm{P} 60-80,{ }^{++}{ }^{++} p<0.0005$ vs BLA HFS, LSD post hoc test). $\boldsymbol{d}$, Summary of the recording and stimulating sites from another cohort of naive rats in which the vHipp HFS was delivered prior to BLA induction of PFCLTP.e, PFC recordings from the P60-80 $(n=7)$ age group revealed an inhibitory effect of vHipp HFS on the expression of BLA-LTP. Such inhibitory control by the vHipp is lacking in the PFC of P30 - 40 rats $(n=6)$. $\boldsymbol{f}$, Summary of the PFC response obtained $30-40$ min post-HFS period. Two-way ANOVA revealed a main effect of input stimulation $\left(F_{(1.22)}=32.6, p<0.0005\right)$, a main effect of age $\left(F_{(1,22)}=37, p<0.0005\right)$, and an age $\times$ input stimulation interaction

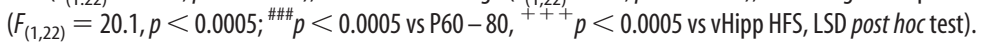
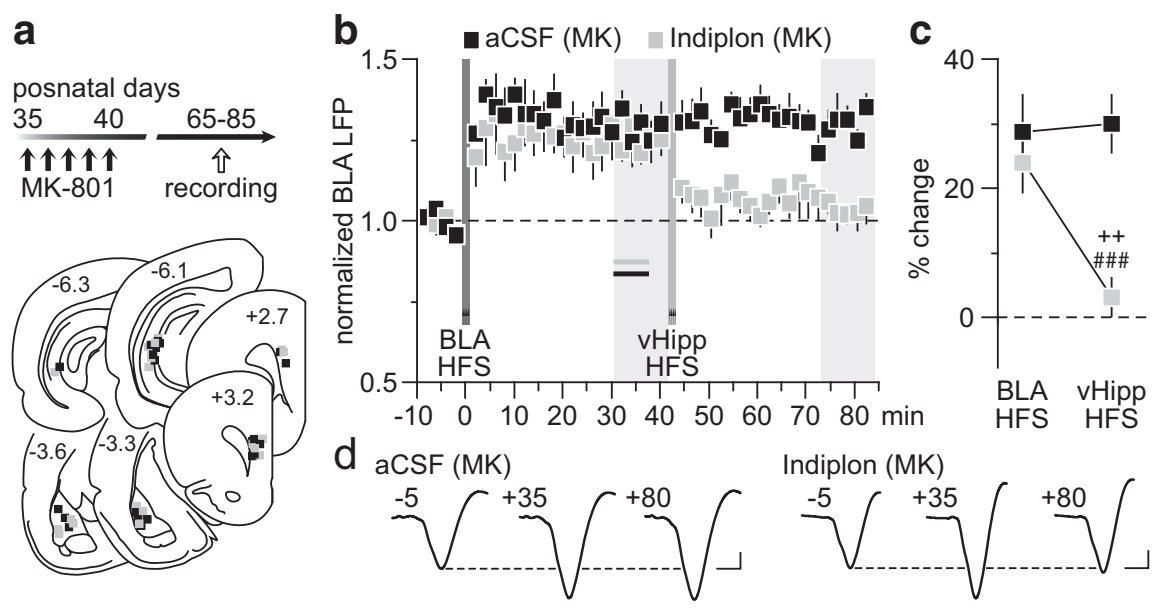

Figure 7. Reversal effect of the $\mathrm{GABA}_{A} \alpha 1$-positive allosteric modulator indiplon on PFC plasticity. $\boldsymbol{a}$, Timeline of the experimental design and summary of the recording sites (PFC) and stimulating sites (BLA and vHipp). $\boldsymbol{b}$, Local PFC infusion of indiplon before vHipp HFS ( $n=7)$ was sufficient to restore the normal vHipp depotentiation of BLA-dependent LTP in MK-801-treated rats (MK). This reversal effect was not observed when aCSF alone was delivered into the PFC $(n=7)$. $c$, Analysis of the mean PFC response obtained at $30-40$ min post-HFS revealed a main effect of infusion $\left(F_{(1,24)}=11.3, p<0.005\right)$, a main effect of input stimulation $\left(F_{(1,24)}=4.5, p<0.05\right)$, and an infusion $\times$ input stimulation interaction $\left(F_{(1,24)}=5.8, p<0.05\right.$; ${ }^{\# \#} p<0.0005$ vs aCSF, ${ }^{++} p<0.005$ vs BLA HFS, LSD post hoc test). d, Example traces of the BLA-evoked PFC response recorded at 5 min pre-BLA HFS (-5), 35 min post-BLA HFS (+35), and 40 min post-vHipp HFS ( +80$)$. Calibration: $4 \mathrm{mV}, 20 \mathrm{~ms}$. cannot account for the age-dependent effects of MK-801 exposure observed here. In fact, recordings from P105-125 rats that received MK-801 treatment during early adolescence revealed an impaired vHipp-dependent depotentiation of the BLAdriven LTP (Fig. $5 c, d$ ) that resembled the P65-85 PFC recordings from the same age and treatment group (Fig. 3). Similarly, vHipp HFS effectively blocked the expression of BLA-induced LTP in both saline solution- and MK-801-treated groups (Fig. 5e). Notably, such inhibitory control by the vHipp is lacking in the P105-125 PFC of early adolescent MK801-treated rats (Fig. 5e,f). Together, these results point towards an agedependent effect of MK-801 on PFC plasticity that endures throughout adulthood, in particular within the vHipp-to-PFC pathway and its control over BLA drive.

As the refinement of PFC plasticity during adolescence is input specific (Caballero et al., 2014), the lack of vHipp control of BLA drive in the PFC observed after early adolescent MK-801 treatment could be due to an impaired functional maturation of the hippocampal-to-PFC pathway. Therefore, we asked how vHipp regulation of BLA transmission in the PFC changes during the transition to adulthood in two distinct age groups of naive rats: $\mathrm{P} 30-40$ versus $\mathrm{P} 60-80$ (Fig. 6). Results show that while hippocampal HFS resulted in depotentiation of the BLAevoked LTP in the P60-80 PFC, such a modulation was lacking when tested in the P30-40 age group (Fig. 6b,c). This deficient hippocampal inhibition (Fig. 6b) is indistinguishable from that seen in the adult PFC of early adolescent MK-801exposed rats (Fig. 3). Similarly, vHipp HFS also blocked the expression of BLAinduced LTP only in the P60-80 age group (Fig. 6d,e), whereas the vHipp-insensitive BLA-dependent LTP observed in the P30 - 40 PFC resembles that found after early adolescent MK-801 treatment (Fig. 4). Together, these data indicate that transient NMDAR blockade during early adolescence impairs the functional maturation of the vHipp-dependent inhibitory control of BLA drive in the PFC, a developmental disruption that endures into adulthood.

Based upon our recent finding of a local GABAergic component mediating LTD in the PFC (Caballero et al., 2014), we asked whether local infusion of the $\mathrm{GABA}_{\mathrm{A}} \alpha 1$-positive allosteric modulator indiplon (Petroski et al., 2006; Thomases et al., 2013) would be sufficient to restore the vHipp inhibitory control of BLA drive 
that is lacking in the PFC of early adolescent MK-801-treated rats. To this end, we conducted recordings from adult rats that received MK-801 during early adolescence and found that a single PFC infusion of indiplon before the vHipp HFS (for details, see Materials and Methods) effectively enabled the vHipp-dependent depotentiation of BLA-evoked LTP, whereas aCSF injection did not (Fig. 7). In fact, the pattern of depotentiation observed in the indiplon-treated PFC of MK-801exposed rats resembled that seen in saline controls. A similar permissive effect of indiplon was observed when the vHipp HFS preceded that of the BLA (Fig. 8). Typically, vHipp HFS does not prevent the induction of prefrontal LTP by the BLA in early adolescent MK-801-treated rats (Fig. 4). However, when indiplon was delivered into the PFC before the vHipp HFS, a complete reinstatement of the normal vHipp-dependent inhibition of BLA-LTP expression emerges (Fig. 8). Interestingly, such inhibition of the BLAmediated expression of LTP in the PFC was lacking if the infusion of indiplon occurred after the vHipp HFS (Fig. 8), suggesting that the inhibitory control enabled by the enhanced $\mathrm{GABA}_{\mathrm{A}}$ function in the PFC is input specific and dependent on plasticity mechanisms at local GABAergic synapses. Collectively, these results point to a mechanistic link between the impaired hippocampal regulation of BLA transmission in the PFC and local deficits in GABAergic function that endure through adulthood as a result of early adolescent MK-801 exposure.

\section{Discussion}

In the present study, we have found that the age-dependent and input-specific dysregulation of PFC plasticity (a switch from vHipp-driven LTD to LTP) resulting from transient early adolescent NMDAR blockade (i.e., MK-801) endures through adulthood due to a developmental impairment of local prefrontal GABAergic function. Our data also show that despite the normal PFC response to BLA stimulation, the distinctive vHipp inhibition of BLA-dependent plasticity in the PFC is lacking after early adolescent MK-801 treatment. Such deficient hippocampal regulation of PFC plasticity resembles that seen in drug-naive juveniles, an impairment that can be fully restored by PFC infusion of the $\mathrm{GABA}_{\mathrm{A}} \alpha 1$-positive allosteric modulator indiplon. Although we are not aware of any targets of indiplon other than the ones currently described (i.e., $\mathrm{GABA}_{\mathrm{A}} \alpha 1$ ), its effects in the PFC appear to be limited to $\mathrm{GABA}_{\mathrm{A}}$-mediated processes without altering $\mathrm{GABA}_{\mathrm{A}}$-independent events such as the evoked plasticity elicited from the BLA (Figs. 7, 8) and the response to vHipp $10 \mathrm{~Hz}$ stimulation (Thomases et al., 2013). Altogether, these results indicate that the normal developmental acquisition of vHipp inhibitory control of BLA transmission in the PFC is impaired by early adolescent MK-801 exposure as a consequence of diminished local prefrontal GABAergic function.

While the functional connectivity from both vHipp and BLA inputs to the PFC continues to mature into early adulthood (Benes, 1989; Cunningham et al., 2002; Thomases et al., 2013; Caballero et al., 2014), only the one arising from the vHipp is

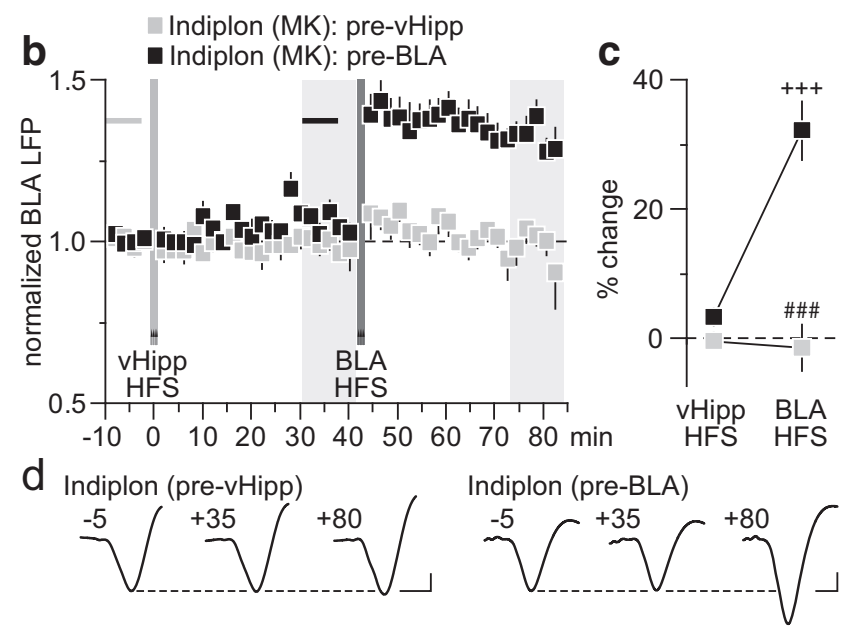

Figure 8. Local prefrontal facilitation of $\mathrm{GABA}_{\mathrm{A}} \alpha 1$ function restores the normal vHipp inhibitory control of $\mathrm{BLA}$ transmission. $\boldsymbol{a}$ Timeline of the experimental design and summary of the recording sites (PFC) and stimulating sites (BLA and vHipp). $\boldsymbol{b}$, Local PFC

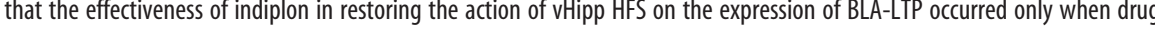
Summary of the mean PFC response obtained at 30-40 min post-HFS. Two-way ANOVA revealed a main effect of infusion $\left(F_{(1,22)}=43.4, p<0.0005\right)$, a main effect of input stimulation $\left(F_{(1,22)}=19.99, p<0.0005\right)$, and an infusion $\times$ input stimulation

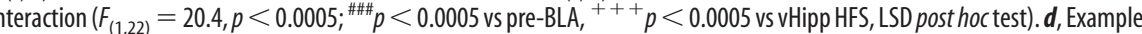
traces of the BLA-evoked PFC response recorded at 5 min pre-vHipp HFS $(-5), 35$ min post-vHipp HFS $(+35)$, and 40 min post-BLA

susceptible to the developmental disturbance induced by MK801 during early adolescence. In the PFC, such disruption is manifested by a shift from the normal vHipp-driven LTD to an LTP indistinguishable from that attained after local PFC infusion of the $\mathrm{GABA}_{\mathrm{A}}$ receptor antagonist picrotoxin. This suggests that blockade of NMDAR transmission during early adolescence impairs the functional recruitment of prefrontal GABAergic circuits by the vHipp that is critical for the expression of input-specific LTD in the adult PFC (Caballero et al., 2014). Interestingly, our data also indicate that the enduring PFC dysregulation resulting from early adolescent MK-801 treatment does not affect the expression of afferent-driven/NMDAR-mediated LTP that typically appears in the PFC after P50 (Flores-Barrera et al., 2014). Thus, the selective impairment of LTD observed after early adolescent MK-801 exposure emerges as an input-specific deficit of vHipp-dependent plasticity because it relies upon the activation of a functionally mature GABAergic network in the PFC (Caballero et al., 2014). In this regard, any developmental deficit of prefrontal GABAergic function will selectively disrupt the response to vHipp transmission in an input-specific manner.

In addition to the impaired LTD, the vHipp inhibition of the BLA-evoked response in the PFC is also absent after early adolescent MK-801 exposure. In the normal adult PFC, inputs from the vHipp possess the functional capacity to reset and inhibit the LTP state of BLA transmission. Although the precise cellular mechanism underlying such inhibitory control is not fully understood (see below), it is clear from our recent (Thomases et al., 2013) and present studies that transient NMDAR blockade during early adolescence is sufficient to induce an enduring prefrontal deficit that disables the normal hippocampal regulation of BLAdependent plasticity in the PFC. Interestingly, the inability of the vHipp to diminish the gain of BLA drive is strikingly similar to that seen in the immature PFC of drug-naive juvenile rats, a disruption that was not observed when MK-801 exposure occurred in adulthood. Together, these results suggest that the 
functional acquisition of hippocampal inhibitory control of BLA transmission in the PFC is developmentally regulated and susceptible to transient attenuation of NMDAR signaling during adolescence in an age-dependent manner.

Our recent study indicates that the expression of vHippdependent LTD in the PFC requires local $\mathrm{GABA}_{\mathrm{A}}$ transmission, whereas plasticity elicited from the BLA does not (Caballero et al., 2014). Such input-specific GABAergic contribution of PFC output plasticity cannot be explained by a differential innervation of vHipp and BLA afferents onto GABAergic interneurons and pyramidal neurons (Bacon et al., 1996; Carr and Sesack, 1996; Gabbott et al., 2002; Tierney et al., 2004). Instead, our studies suggest a distinct functional recruitment of PFC GABAergic interneurons by the vHipp that cannot be elicited by BLA inputs. Although the precise synaptic locus of this input-specific modulation remains to be determined, our data are consistent with a form of LTP plasticity occurring at prefrontal GABAergic synapses that contributes to both the expression of vHippdependent LTD and the inhibitory control this pathway exerts over BLA transmission in the PFC. Indeed, strengthening of PFC GABAergic function via local infusion of indiplon completely restored the vHipp-dependent inhibitory regulation of BLA transmission that is lacking in rats exposed to MK-801 during early adolescence. Notably, this inhibitory control of PFC plasticity occurred only when indiplon was delivered before (not after) HFS of the vHipp. Together, these results point to a lack of prefrontal GABAergic plasticity as a key mechanism by which transient blockade of NMDA transmission during adolescence disrupts PFC processing of afferent information.

The age- and GABAergic-dependent dysregulation of PFC plasticity observed here with MK-801 is expected to compromise a variety of cognitive functions, including working memory, attention, and the control of impulsive behavior, all of which require proper integration of afferent information originating from the vHipp and BLA by the PFC (Phillips and LeDoux, 1992; Ishikawa and Nakamura, 2003; Sierra-Mercado et al., 2011; Chudasama et al., 2012; Gilmartin et al., 2012; Sotres-Bayon et al., 2012). In addition, behavioral studies using fear-conditioning paradigms have revealed that BLA (Anglada-Figueroa and Quirk, 2005; Sierra-Mercado et al., 2006; Gilmartin et al., 2012) and vHipp (Milad et al., 2007; Sotres-Bayon et al., 2012) inputs to the PFC control the expression and extinction of conditioned fear responses, respectively. Such discrete behaviors are thought to be related to the well established role of the vHipp and the BLA in regulating PFC plasticity (Laroche et al., 2000; Maroun and Richter-Levin, 2003; Caballero et al., 2014). Our data present a scenario in which the gain of BLA transmission in the PFC depends on the functional control of GABA plasticity by the vHipp, thus tuning prefrontal processing of emotionally salient stimuli and controlling behavioral inhibition. A disruption of these processes could emerge as an inappropriate emotional response to neutral stimuli as occurs in many psychiatric disorders including schizophrenia (Benes, 2010), in which deficits in PFC GABAergic interneurons continue to be one of the most common findings (Gonzalez-Burgos et al., 2011; Lewis et al., 2012). Importantly, the fact that PFC plasticity can be normalized by local upregulation of $\mathrm{GABA}_{\mathrm{A}} \alpha 1$ transmission suggests that a pharmacological strengthening of the vHipp inhibitory control of prefrontal output could restore proper PFC functionality in individuals with a developmentally immature/attenuated prefrontal GABAergic system. Future studies are warranted to test this hypothesis.

In summary, the functional maturation of GABAergic plasticity in the PFC requires sustained NMDA transmission during adolescence. As such, any genetic and environmental factors capable of disrupting NMDAR signaling (Do et al., 2009; Kantrowitz and Javitt, 2010) during adolescence are expected to cause long-lasting impairments of prefrontal GABAergic plasticity and subsequent input-specific dysregulation of PFC output by the vHipp.

\section{References}

Anglada-Figueroa D, Quirk GJ (2005) Lesions of the basal amygdala block expression of conditioned fear but not extinction. J Neurosci 25:96809685. CrossRef Medline

Bacon SJ, Headlam AJ, Gabbott PL, Smith AD (1996) Amygdala input to medial prefrontal cortex (mPFC) in the rat: a light and electron microscope study. Brain Res 720:211-219. CrossRef Medline

Benes FM (1989) Myelination of cortical-hippocampal relays during late adolescence. Schizophr Bull 15:585-593. CrossRef Medline

Benes FM (2010) Amygdalocortical circuitry in schizophrenia: from circuits to molecules. Neuropsychopharmacology 35:239-257. CrossRef Medline

Best JR, Miller PH (2010) A developmental perspective on executive function. Child Dev 81:1641-1660. CrossRef Medline

Caballero A, Thomases DR, Flores-Barrera E, Cass DK, Tseng KY (2014) Emergence of GABAergic-dependent regulation of input-specific plasticity in the adult rat prefrontal cortex during adolescence. Psychopharmacology (Berl) 231:1789-1796. CrossRef Medline

Carr DB, Sesack SR (1996) Hippocampal afferents to the rat prefrontal cortex: synaptic targets and relation to dopamine terminals. J Comp Neurol 369:1-15. CrossRef Medline

Casey BJ, Giedd JN, Thomas KM (2000) Structural and functional brain development and its relation to cognitive development. Biol Psychol 54: 241-257. CrossRef Medline

Chudasama Y, Doobay VM, Liu Y (2012) Hippocampal-prefrontal cortical circuit mediates inhibitory response control in the rat. J Neurosci 32: 10915-10924. CrossRef Medline

Cunningham MG, Bhattacharyya S, Benes FM (2002) Amygdalo-cortical sprouting continues into early adulthood: implications for the development of normal and abnormal function during adolescence. J Comp Neurol 453:116-130. CrossRef Medline

Do KQ, Cabungcal JH, Frank A, Steullet P, Cuenod M (2009) Redox dysregulation, neurodevelopment, and schizophrenia. Curr Opin Neurobiol 19:220-230. CrossRef Medline

Flores-Barrera E, Thomases DR, Heng LJ, Cass DK, Caballero A, Tseng KY (2014) Late adolescent expression of GluN2B transmission in the prefrontal cortex is input-specific and requires postsynaptic protein kinase $\mathrm{A}$ and D1 dopamine receptor signaling. Biol Psychiatry 75:508-516. CrossRef Medline

Gabbott PL, Warner TA, Busby SJ (2006) Amygdala input monosynaptically innervates parvalbumin immunoreactive local circuit neurons in rat medial prefrontal cortex. Neuroscience 139:1039-1048. CrossRef Medline

Gabbott P, Headlam A, Busby S (2002) Morphological evidence that CA1 hippocampal afferents monosynaptically innervate PV-containing neurons and NADPH-diaphorase reactive cells in the medial prefrontal cortex (Areas 25/32) of the rat. Brain Res 946:314-322. CrossRef Medline

Gilmartin MR, Kwapis JL, Helmstetter FJ (2012) Trace and contextual fear conditioning are impaired following unilateral microinjection of muscimol in the ventral hippocampus or amygdala, but not the medial prefrontal cortex. Neurobiol Learn Mem 97:452-464. CrossRef Medline

Gogtay N, Vyas NS, Testa R, Wood SJ, Pantelis C (2011) Age of onset of schizophrenia: perspectives from structural neuroimaging studies. Schizophr Bull 37:504-513. CrossRef Medline

Gonzalez-Burgos G, Fish KN, Lewis DA (2011) GABA neuron alterations, cortical circuit dysfunction and cognitive deficits in schizophrenia. Neural Plast 2011:723184. CrossRef Medline

Ishikawa A, Nakamura S (2003) Convergence and interaction of hippocampal and amygdalar projections within the prefrontal cortex in the rat. J Neurosci 23:9987-9995. Medline

Kantrowitz JT, Javitt DC (2010) N-methyl-d-aspartate (NMDA) receptor dysfunction or dysregulation: the final common pathway on the road to schizophrenia? Brain Res Bull 83:108-121. CrossRef Medline

Kessler RC, Amminger GP, Aguilar-Gaxiola S, Alonso J, Lee S, Ustün TB (2007) Age of onset of mental disorders: a review of recent literature. Curr Opin Psychiatry 20:359-364. CrossRef Medline 
Laroche S, Davis S, Jay TM (2000) Plasticity at hippocampal to prefrontal cortex synapses: dual roles in working memory and consolidation. Hippocampus 10:438-446. CrossRef Medline

Lewis DA, Curley AA, Glausier JR, Volk DW (2012) Cortical parvalbumin interneurons and cognitive dysfunction in schizophrenia. Trends Neurosci 35:57-67. CrossRef Medline

Maroun M, Richter-Levin G (2003) Exposure to acute stress blocks the induction of long-term potentiation of the amygdala-prefrontal cortex pathway in vivo. J Neurosci 23:4406-4409. Medline

Milad MR, Wright CI, Orr SP, Pitman RK, Quirk GJ, Rauch SL (2007) Recall of fear extinction in humans activates the ventromedial prefrontal cortex and hippocampus in concert. Biol Psychiatry 62:446-454. CrossRef Medline

Paus T, Keshavan M, Giedd JN (2008) Why do many psychiatric disorders emerge during adolescence? Nat Rev Neurosci 9:947-957. CrossRef Medline

Petroski RE, Pomeroy JE, Das R, Bowman H, Yang W, Chen AP, Foster AC (2006) Indiplon is a high-affinity positive allosteric modulator with selectivity for alphal subunit-containing $\mathrm{GABA}_{\mathrm{A}}$ receptors. J Pharmacol Exp Ther 317:369-377. CrossRef Medline

Phillips RG, LeDoux JE (1992) Differential contribution of amygdala and hippocampus to cued and contextual fear conditioning. Behav Neurosci 106:274-285. CrossRef Medline

Sierra-Mercado D Jr, Corcoran KA, Lebrón-Milad K, Quirk GJ (2006) Inactivation of the ventromedial prefrontal cortex reduces expression of conditioned fear and impairs subsequent recall of extinction. Eur J Neurosci 24:1751-1758. CrossRef Medline

Sierra-Mercado D, Padilla-Coreano N, Quirk GJ (2011) Dissociable roles of prelimbic and infralimbic cortices, ventral hippocampus, and basolateral amygdala in the expression and extinction of conditioned fear. Neuropsychopharmacology 36:529-538. CrossRef Medline

Sotres-Bayon F, Sierra-Mercado D, Pardilla-Delgado E, Quirk GJ (2012) Gating of fear in prelimbic cortex by hippocampal and amygdala inputs. Neuron 76:804-812. CrossRef Medline

Thomases DR, Cass DK, Tseng KY (2013) Periadolescent exposure to the NMDA receptor antagonist MK-801 impairs the functional maturation of local GABAergic circuits in the adult prefrontal cortex. J Neurosci 33:26-34. CrossRef Medline

Tierney PL, Dégenètais E, Thierry AM, Glowinski J, Gioanni Y (2004) Influence of the hippocampus on interneurons of the rat prefrontal cortex. Eur J Neurosci 20:514-524. CrossRef Medline 